\title{
Garcinia cambogia and diabetes mellitus: a lack of evidence?
}

\begin{abstract}
Diabetes Mellitus is a disease characterized by hyperglycemia resulting from defects in insulin secretion. Garcinia Cambogia is an Asian plant used commonly as nutritional supplement for weight loss.
\end{abstract}

Objective: The purpose of this study is to perform a mini review about Garcinia Cambogia consumption and its relationship with DM condition in humans.

Methods: Studies were searched in Pubmed, Web of Science and Scopus databases and 62 articles were identified and reviewed in an integrative method. After a criterial analysis, 3 articles were included on this mini review.

Results: Two studies found that Garcinia Cambogia in association with Glucomannan had a positive association related to comorbidities as Diabetes Mellitus and its markers (glycemia, triglycerides, cholesterol). However, a case report showed an adverse effect on a high dose Garcinia Cambogia consumption.

Conclusion: It is possible that Garcinia Cambogia consumption influences Diabetes Mellitus condition, although more studies are necessary for higher efficacy understanding.

Keywords: garcinia cambogia, diabetes mellitus, mini review
Volume 6 Issue 3 - 202

\author{
Rafaella Câmara Rocha Menezes,' Juliana \\ Crystal Schumacher, ${ }^{2}$ Anna Caroline \\ Cristofoli Bertoletti, ${ }^{3}$ Larissa Slongo \\ Faccioli, ${ }^{3}$ Jordana Boeira Freitas, ${ }^{2}$ Isabella \\ Rosa da Mata, ${ }^{3}$ Kathleen Kruger Peres, ${ }^{3}$ \\ Carine Fabiana Saul, ${ }^{2}$ Caroline Joana \\ Kuyven, ${ }^{2}$ Simone Morelo Dal Bosco ${ }^{2,3}$ \\ 'Nutrition Department, Universidade Federal do Rio Grande \\ do Sul, Brazil \\ 2Undergraduate Program in Nutrition, Universidade Federal de \\ Ciências da Saúde de Porto Alegre, Brazil \\ ${ }^{3}$ Graduate Program in Nutrition Sciences, Universidade Federal \\ de Ciências da Saúde de Porto Alegre, Brazil
}

Correspondence: Simone Morelo Dal Bosco, PhD,

Department of Nutrition, Universidade Federal de Ciências da Saúde de Porto,Alegre (UFCSPA). 245, Sarmento Leite. Porto Alegre, RS - Brazil, Tel 3303-8700,

Emailsimonedb@ufcspa.edu.br
Abbreviations: GC, garcinia cambogia; GNN, glucomannan, garcinia cambogia and diabetes mellitus; DM, diabetes mellitus

\section{Introduction}

Diabetes Mellitus (DM) is a disease characterized by hyperglycemia resulting from defects in insulin secretion. The types of development of diabetes range from deficiency of insulin ensues consequent to pancreatic beta cell destruction to abnormalities that result in resistance insulin.

The presence of hyperglycemia marked features symptoms as polyuria, polydipsia, weight loss, polyphagia, and blurred vision. Other than that, the consequences of diabetes affect nearly every tissue of the body and diabetes is a leading cause of cardiovascular. Acute complications of uncontrolled diabetes are ketoacidosis or the nonketotic hyperosmolar syndrome; chronic complications include potential loss of vision, renal failure peripheral neuropathy with risk of foot ulcers, amputations and autonomic neuropathy causing gastrointestinal, genitourinary, and cardiovascular symptoms and sexual dysfunction. Increased incidence of cardiovascular morbidity and mortality and abnormalities of lipoprotein metabolism are often found in people with diabetes. ${ }^{2}$

The garcinia cambogia (GC) seeds are used to treat various diseases, abdominal pain, food allergies, arthritis, diarrhea, dysentery and wounds, as well as can be used as a hypoglycemic agent and in infections. The use of herbal medicines has gained acceptance in recent years, as they are of natural origin and of low cost. Herbal medicines have been used for a long time in some cultures, such as Asian and Indian, and are spread around the world as an alternative. The use of herbal medicines has gained acceptance in recent years, as they are of natural origin and of low cost. It is known that these herbal medicines have been used for a long time in some cultures, such as Asian and
Indian. One of these agents is $\mathrm{GC}$, an extract from an Asian plant that has been used as a nutritional supplement to control weight. ${ }^{3,4}$

Considering the numerous benefits of GC and the importance of diabetes: the aim of this study was to perform a mini review about GC consumption and its relationship with DM condition in humans.

\section{Methods}

This is a mini review, with an integrative method to review the literature carried out in Pubmed, Scopus and Web of Science in April 2021. The descriptors used were "garcinia cambogia" and "diabetes mellitus", both identified as Mesh Terms (Medical Subject Headings). Articles included were in indexed journals, considering languages English, Portuguese, and Spanish. The research question for this review was: "Is Garcinia Cambogia consumption related to an improvement in diabetes mellitus condition in humans?". In total, 62 articles were found in databases, 11 considered for full-reading and, in the phase, 2 articles were excluded for language and 1 was not found. After criterial analysis, 3 articles were used to guide this review. The selected articles were read in a detailed and complete manner and were discussed with other articles with consistency in the results found.

\section{Results}

In a previous study, Maia-Landim et al. ${ }^{5}$ found that the treatment using GC combined with Glucomannan (GNN) (GC and GNN, 500 $\mathrm{mg}-2$ capsules/day) there was a reduction in weight (independent age, sex or previously reported condition), rate of viceral fat and fat mass and an increase in the basal metabolic rate in overweight or obese individuals during 6 months of treatment. Compared to the baseline, the reduction in triglyceride, cholesterol and glucose levels has also been shown to be effective. 
In a pre-experimental study, Maia-Landim et al. ${ }^{5}$ had a result that obese or overweight adults used Garcinia Cambogia and Glucomannan $(1 \mathrm{~g} /$ day $)$ for 3 and 6 months. The results were independent of age, sex or the presence of chronic non-communicable diseases (dyslipidemia, hypertension, or type 2 diabetes mellitus), these being presented by a reduction in fat mass, visceral fat, lipids, blood glucose, weight loss and also an increase in basal metabolic rate.

In a case reported by Bystrak et al. ${ }^{6}$ a woman consumed GC via oral three times a day (1.400-1.440 mg/day) and an unknown dietetic supplement of Irvingia gabonenses. Besides that, the patient had a series of comorbidities, using numerous medications. Furthermore, she was diabetic, using high-dose insulin. As a result, the drug interaction between the use of Garcinia Cambogia and the other drugs led to a serious adverse effect, with pancreatitis and diabetic ketoacidosis.

\section{Discussion}

The double-blind randomized study carried out by Vasques et al. ${ }^{7}$ presented an evaluation of pharmacotherapeutic efficacy of the standardized extracts of G. cambogia (52.4\% HCA) and A. konjac ( $94.9 \%$ of glucomannan) as a therapeutic form in obesity. In the experiment, 32 people were treated with GC and GNN for more than 12 weeks a daily dose $(2.4 \mathrm{~g} ; 1.5 \mathrm{~g}$, respectively). In this research, lower levels of total cholesterol $(-32.0 \pm 35.1 \mathrm{mg} / \mathrm{dL})$ and LDL-c $(-28.7 \pm 32.7 \mathrm{mg} / \mathrm{dL})$ were found in the intervention group, data that corroborate with the findings Maia-Landim et al. ${ }^{5}$

Opposing the results of Maia-Landim et al. ${ }^{5}$ the systematic review by Pittler and Ernst ${ }^{8}$ brought information regarding the indiscriminate use of dietary supplements as aids in reducing weight and their use without prescription, which is an unconvincing use. However, the study by Maia-Landim et al. ${ }^{5}$ mentions the use of GC and GNN as therapeutic pharmaceuticals, also known as possible herbal medicines.

In the case report by Bystrak et al. ${ }^{6}$ the reported unfavorable effects were considered rare in human toxicity analyzes. In Márquez's research, ${ }^{9}$ studies using GC extracts in humans and animals have been evaluated to assess efficacy and toxicity. The data were collected and synthesized for comparability and to be able to assess whether there is evidence to consider the CG safe to consume. In studies whose purpose was to assess toxicity and safety, no increase in mortality or possible significant toxicity has been reported when conducted with animal models. ${ }^{10}$

\section{Conclusion}

The consumption of Garcinia Cambogia associated with a good quality of life can improve the health of people with Diabetes Mellitus.
It is necessary to be very careful in consumption and must have medical and nutritional guidance. However, further studies should be carried out to evaluate the effectiveness of the CG, since there is still a lack of evidence regarding its mechanism of action.

\section{Acknowledgments}

None.

\section{Conflicts of interest}

The authors declare that there is no conflict of interest.

\section{References}

1. American diabetes association: standards of medical care in diabetes 2020. Diabetes Care. 2020;43supp11:S1-S2.

2. Schmidt A. Highlighting diabetes mellitus: the epidemic continues. Arterioscler Thromb Vasc Biol. 2018;38(1):e1-e8.

3. Maia-Landim A, Lancho C, Poblador M, et al. Garcinia cambogia and Glucomannan reduce weight, change body composition and ameliorate lipid and glucose blood profiles in overweight/obese patients. Jornal of Herbal Medicine. 2021;26:100424.

4. Chen T-H, Tsai M-J, Fu Y-S, et al. The Exploration of natural compounds for anti-diabetes from distinctive species garcinia linii with comprehensive review of the garcinia family. Biomolecules. 2019;9(11):641.

5. Maia-Landin A, Ramírez J, Lancho C, et al. Long-term effects of Garcinia cambogia/Glucomannan on weight loss in people with obesity. 2018;18(1):26.

6. Bystrak T, Cervera-Hernandez E, Reddy N, et al. Garcinia cambogia, diabetic ketoacidosis and pancreatitis. $R$ I Med J. 2013;100(10):48-50.

7. Vasques, A, Rossetto, S, Halmenschlager, G, et al. Evaluation of the pharmacotherapeutic efficacy of Garcinia cambogia plus Amorphophallus konjac for the treatment of obesity. Phytotherapy Research. 2008;22(9):1135-1140.

8. Pittler, M, Ernst, E. Dietary supplements for body-weight reduction: a systematic review. The American journal of clinical nutrition. 2004;79(4):529-536.

9. Márquez F, Babio N, Bulló M, et al. Evaluation of the safety and efficacy of hydroxycitric acid or Garcinia cambogia extracts in humans. Crit Rev Food Sci Nutr. 2012;52(7):585-94.

10. LIN4, FTO and Trp64Arg polymorphisms. BMC Complement Altern Med. 2018:18-26. 